\title{
OPEN Mortality trends in an ambulatory multidisciplinary heart failure unit from 2001 to 2018
}

\author{
Giosafat Spitaleri ${ }^{1}$, Josep Lupón ${ }^{1,2,3}$, Mar Domingo ${ }^{1}$, Evelyn Santiago-Vacas ${ }^{1}$, Pau Codina ${ }^{1}$, \\ Elisabet Zamora ${ }^{1,2,3}$, Germán Cedie ${ }^{1,2}{ }^{2}$, Javier Santesmases ${ }^{1,2}$, Crisanto Diez-Quevedo ${ }^{1}$, \\ Maria Isabel Troya ${ }^{1}$, Maria Boldo ${ }^{1}$, Salvador Altmir ${ }^{1}$, Nuria Alonso ${ }^{1}$, Beatriz González ${ }^{1}$, \\ Julio Núñez ${ }^{3,4,5}$ \& Antoni Bayes-Genis ${ }^{1,2,3,6}$
}

To assess mortality trends at 1 and 3 years from 2001 to 2018 in a real-life cohort of HF outpatients from different etiologies with depressed and preserved LVEF. A total of 2368 consecutive patients with $\mathrm{HF}$ (mean age $66.4 \pm 12.9$ years, $71 \%$ men, $15.4 \%$ with preserved LVEF) admitted to a HF clinic from August 2001 to September 2018 were included in the study. Patients were divided into five quintiles (Q) according to the period of admission. Trends for all-cause and cardiovascular mortality from Q1 to 05 were assessed by linear regression. Patients with $\mathrm{LVEF}<50 \%$ had a progressive decrease in the rates of all-cause and cardiovascular death at 1 year (12.1\% in 01 to $6.5 \%$ in $05, p=0.003$; and $8.4 \%$ in 01 to $3.8 \%$ in $Q 5, p=0.007$, respectively) and 3 years ( $30.5 \%$ in 01 to $17.0 \%$ in $05, p=0.003$; and $23.9 \%$ in 01 to $9.8 \%$ in $05, p=0.003$, respectively). These trends remained significant after adjusting for clinical characteristics and risk. No significant trend in mortality was observed in patients with $\mathrm{LVEF} \geq 50 \%$. In a cohort of real-life ambulatory patients with $\mathrm{HF}$, mortality progressively declined in patients with LVEF $<50 \%$, but the same trend was not observed in patients with preserved LVEF.

Over the last three decades, since the introduction of angiotensin-converting enzyme inhibitors (ACEIs), there has been a progressive reduction in mortality rates across trials testing new therapies in patients with heart failure $(\mathrm{HF})$ and reduced left ventricular ejection fraction $(\mathrm{LVEF})^{1-10}$. Therefore, current guidelines recommend the use of drugs inhibiting the neurohormonal axis and devices, such as implantable cardioverter defibrillator (ICD) and cardiac resynchronization therapy (CRT), to prolong survival in patients with HF and reduced LVEF $(\mathrm{HFrEF})^{11}$. Despite advances in HF management, which also include the implementation of HF clinics and specialized HF teams, the prognosis is still poor in the real-life setting. Data from registries indicate that, regardless of ejection fraction, mortality in HF patients can be as high as $75 \%$ at 5 years ${ }^{12}$, a rate that is significantly higher than in clinical trials, in which patients are usually younger and have fewer comorbidities. In addition, no improvement in survival has been observed across trials enrolling patients with HF with preserved LVEF (HFpEF). Furthermore, despite encouraging results from recent studies, no convincing evidence-based therapy for HFpEF has been identified ${ }^{13,14}$. Thus, mortality from HF remains a challenge in real-world practice.

In the present study, we assessed mortality trends at 1 and 3 years, from 2001 to 2018, in a real-life cohort of HF outpatients with depressed and preserved LVEF.

\section{Methods}

Study population and outcomes. All consecutive ambulatory patients admitted to a structured multidisciplinary HF clinic at a university hospital between August 2001 and September 2018, regardless of aetiology, were considered for the study. During the 19-year study period, the clinical pathways and referral geographic area, covering 850,000 inhabitants in the northern Barcelona Metro Area, remained stable. Patients were referred to the HF clinic mostly by the Cardiology or Internal Medicine Departments, and to a lesser extent by the Emergency Department or other hospital departments. The criteria for referral to the HF clinic were HF

\footnotetext{
${ }^{1}$ Heart Failure Clinic and Cardiology Service, University Hospital Germans Trias I Pujol, Badalona, Spain. ${ }^{2}$ Department of Medicine, Universitat Autonoma de Barcelona, Barcelona, Spain. ${ }^{3} \mathrm{CIBERCV}$, Instituto de Salud Carlos III, Madrid, Spain. ${ }^{4}$ Cardiology Department, Hospital Clínico Universitario, INCLIVA, València, Spain. ${ }^{5}$ Department of Medicine, Universitat de València, València, Spain. ${ }^{6}$ Heart Institute, Hospital Universitari Germans Trias I Pujol, Department of Medicine, Universitat Autonoma de Barcelona, Carretera del Canyet s/n 08916, Badalona, Spain. ${ }^{\square}$ email: abayesgenis@gmail.com
} 
according to the European Society of Cardiology (ESC) guidelines, regardless of aetiology, at least one HF hospitalization, and/or reduced LVEF ${ }^{15,16}$. All patients were seen regularly for follow-up visits at the HF clinic according to their clinical needs and treated according to a unified protocol. Follow-up visits included a minimum of one visit with a nurse every 3 months and one visit with a physician (cardiologist, internist, or family physician) every 6 months, as well as optional visits with specialists in geriatrics, psychiatry, and rehabilitation ${ }^{15,16}$, with the addition of a nephrologist and endocrinologist in recent years.

Patients were divided into quintiles according to the period of admission as follows: quintile 1 (Q1) from August 3, 2001, to August 31, 2004; quintile 2 (Q2) from September 1, 2004, to November 10, 2008; quintile 3 (Q3) from November 11, 2008, to June 22, 2012; quintile 4 (Q4) from June 23, 2012, to October 9, 2015; quintile 5 (Q5) from October 10, 2015, to September 28, 2018. For the purpose of this study, all-cause and cardiovascular death rates were estimated 1 and 3 years after admission in all quintiles. In addition, recurrent HF-related hospitalizations during the same study time periods were assessed. In order to avoid possible bias due to different follow-up times, we calculated the number of HF hospitalizations per 100 patients-years for patients of each admission period. Mortality trends were assessed across the study period in the overall population and in the depressed (a category that includes patients with $\mathrm{LVEF}<50 \%$ ) and preserved (LVEF $\geq 50 \%$ ) subgroups. Fatal events were identified from the patients' health records (including hospital wards, emergency room, and general practitioners) or by contacting their relatives. Data were verified using the databases of the Catalan and Spanish Health Systems. Events were adjudicated by an ad hoc committee (JL, MdeA, BG, and MD; PM resolved the possible discrepancies). No loss of follow-up at 5 years was observed for vital status. For HF-related hospitalizations, 37 patients were lost for hospitalizations data during follow-up due to geographic mobility. These patients were adequately censored at the time of transfer. During the baseline visit, patients provided written consent for the use of their clinical data for research purposes. The study was performed in compliance with the law protecting personal data in accordance with the international guidelines on clinical investigations from the World Medical Association's Declaration of Helsinki. Informed consent was obtained from all participants. The local ethics committee (Research Ethics Committee, Germans Trias i Pujol University Hospital) approved the study (ethic code REGI-UNIC PI-18-037).

Statistical analysis. Categorical variables were expressed as absolute numbers and percentages. Continuous variables were expressed as the mean \pm standard deviation (SD) or median [interquartile range] according to normal or non-normal distributions. Normal distributions were assessed by normal Q to Q plots. Trends in baseline characteristics across quintiles were assessed by the Mantel-Haenszel test of trend for categorical variables and linear regression for continuous variables.

Patients with depressed and preserved LVEF were compared using chi-squared and Fisher's exact test for categorical variables, and the Student's t-test or U Mann-Whitney test for continuous variables, as appropriate. Trends for all-cause and cardiovascular mortality from the first to the last quintile were assessed by linear regression, using the period of admission as the independent variable. To analyse the potential confounding effect of baseline variables on the risk of all-cause and cardiovascular death, two multiple regression models were used. In model 1, stepwise multiple regression analyses, including age, sex, LVEF, NYHA class III-IV, and number of comorbidities (among diabetes, hypertension, anaemia, atrial fibrillation or flutter, chronic obstructive pulmonary disease [COPD]) as covariates with quintile of admission, were performed. In model 2, quintile of admission and Meta-Analysis Global Group in Chronic (MAGGIC) Heart Failure Risk Score (at 1 or 3 years) were included and analysed using standard multiple regression. Time to event curves are presented by quintiles of the period of admission as estimated using the Kaplan-Meier method. Statistical analyses were performed in SPSS 24 (SPSS Inc., Chicago, IL, USA). A two-sided P $<0.05$ was considered significant.

\section{Results}

Baseline characteristics. From August 2001 to September 2018, 2368 patients were admitted to our HF clinic. Of these, 2004 (84.6\%) presented with depressed LVEF and 364 (15.4\%) with preserved LVEF at admission. Table 1 summarizes the baseline demographic and clinical characteristics of patients and treatments during follow-up, showing the differences between the two LVEF groups. Overall, the mean age was $66.4 \pm 12.9$ years, $71 \%$ were males, and the prevalence of ischemic heart disease was $48 \%$. Table 2 shows the data according to period of admission quintiles and LVEF subgroups. Regardless of the LVEF, patients admitted in the latter periods reported less severe NYHA functional class symptoms. In the depressed LVEF subgroup, patients admitted more recently had shorter HF duration, higher LVEF, lower 1- and 3-year death risk by MAGGIC score, but more comorbidities. Conversely, patients with LVEF $\geq 50 \%$ were progressively younger, more often female, and had fewer comorbidities.

Treatment during follow-up. In the depressed LVEF group, a significant trend towards an increase in several life-saving treatments (beta-blockers, mineralocorticoid receptor antagonists, ivabradine, and CRT) was observed across quintiles, whereas the opposite occurred with digoxin. In patients with preserved LVEF, the use of beta-blockers and ICD (of which, more than a half [54.5\%] were implanted in patients with HCM) increased, whereas the use of renin-angiotensin system blockers, loop diuretics, and mineralocorticoid receptor antagonists decreased (Table 2).

Changes in LVEF at 1 year. LVEF at 1 year of follow-up was available in 1321 out of the 2004 patients with depressed LVEF and in 185 out of the 364 patients with preserved LVEF. LVEF at 1 year increased significantly during the study periods in patients with depressed LVEF $(p=0.001)$ while it did not change in patients with preserved LVEF (Table 2). 


\begin{tabular}{|c|c|c|c|c|}
\hline & Total cohort $(\mathrm{n}=\mathbf{2 3 6 8})$ & Depressed EF (n= 2004) & Preserved EF $(n=364)$ & p-value \\
\hline Age, years & $66.4 \pm 12.9$ & $65.9 \pm 12.5$ & $69.1 \pm 14.5$ & $<0.001$ \\
\hline Male & $1681(71.0)$ & $1505(75.1)$ & $176(48.4)$ & $<0.001$ \\
\hline Caucasian & $2298(97.0)$ & $1941(96.9)$ & $357(98.1)$ & 0.42 \\
\hline Aetiology & & & & $<0.001$ \\
\hline Ischaemic heart disease & $1141(48.2)$ & $1084(54.1)$ & $57(15.7)$ & \\
\hline Dilated CM & $345(14.6)$ & $334(16.7)$ & $11(3.0)$ & \\
\hline Hypertensive CM & $219(9.2)$ & $132(6.6)$ & $87(23.9)$ & \\
\hline Alcohol CM & $115(4.9)$ & $111(5.5)$ & $4(1.1)$ & \\
\hline Drug-related CM & $65(2.7)$ & $59(2.9)$ & $6(1.6)$ & \\
\hline Valvular & $215(9.1)$ & $130(6.5)$ & $85(23.4)$ & \\
\hline Hypertrophic CM & $72(3.0)$ & $6(0.3)$ & $66(18.1)$ & \\
\hline Amyloidosis & $13(0.5)$ & $4(0.2)$ & $9(2.5)$ & \\
\hline Other & $183(7.7)$ & $142(7.2)$ & $39(10.7)$ & \\
\hline HF duration, months & $7[1-44]$ & $6[1-40]$ & 13 [3-57] & $<0.001$ \\
\hline NYHA class & & & & $<0.001$ \\
\hline I & $166(7.0)$ & $119(5.9)$ & $47(12.9)$ & \\
\hline II & $1534(64.8)$ & $1356(67.7)$ & $178(48.9)$ & \\
\hline III & $640(27.0)$ & $507(25.3)$ & $133(36.5)$ & \\
\hline IV & $28(1.2)$ & $22(1.1)$ & $6(1.6)$ & \\
\hline LVEF, \% & $35.3 \pm 14.2$ & $30.5 \pm 8.7$ & $61.9 \pm 7.8$ & $<0.001$ \\
\hline LVEDD, $\mathrm{mm}^{*}$ & $59.7 \pm 9.3$ & $61.6 \pm 8.2$ & $48.8 \pm 6.9$ & $<0.001$ \\
\hline LVESD, $\mathrm{mm}^{*}$ & $47.0 \pm 11.2$ & $49.6 \pm 9.6$ & $32.4 \pm 7.5$ & $<0.001$ \\
\hline$\triangle$ LVEF at 1 year $\dagger$ & $7.7 \pm 12.0$ & $8.8 \pm 11.9$ & $-0.2 \pm 9.8$ & $<0.001$ \\
\hline Diabetes & $1019(43.0)$ & $867(43.3)$ & $152(41.8)$ & 0.60 \\
\hline Hypertension & $1505(63.6)$ & $1258(62.8)$ & $247(67.9)$ & 0.06 \\
\hline COPD & $406(17.1)$ & $345(17.2)$ & $61(16.8)$ & 0.83 \\
\hline Anaemia $^{\dagger, a}$ & $1072(45.4)$ & $897(44.9)$ & $175(48.2)$ & 0.24 \\
\hline Renal insufficiency ${ }^{\S, b}$ & $1035(44.1)$ & $850(42.8)$ & $185(51.5)$ & 0.002 \\
\hline Atrial fibrillation or flutter & $511(21.6)$ & $360(18.0)$ & $151(41.5)$ & $<0.001$ \\
\hline Number of comorbidities & $1.9 \pm 1.2$ & $1.8 \pm 1.2$ & $2.2 \pm 1.3$ & $<0.001$ \\
\hline 1-year death risk (MAGGIC score) & $13[8-21]$ & $13^{8-21}$ & $13[6-23]$ & 0.10 \\
\hline 3-year death risk (MAGGIC score) & $32[21-46]$ & 32 [21-46] & $32[16-49]$ & 0.0 .10 \\
\hline $\mathrm{BMI}, \mathrm{kg} / \mathrm{m}^{2 \ddagger}$ & $27[24-30]$ & $26[24-30]$ & $27[25-32]$ & 0.03 \\
\hline Obesity & $622(26.5)$ & $509(25.6)$ & $113(31.4)$ & 0.02 \\
\hline NT-proBNP, ng/L' & $1690[711-4095]$ & $1750[764-4263]$ & $1300[394-3210]$ & 0.02 \\
\hline \multicolumn{5}{|l|}{ Treatments } \\
\hline ACEI/ARB/ARNI & $2040(86.1)$ & $1815(90.6)$ & $225(61.8)$ & $<0.001$ \\
\hline ARNI & $257(10.9)$ & $252(12.6)$ & $5(1.4)$ & $<0.001$ \\
\hline Beta-blocker & $2104(88.9)$ & $1842(91.9)$ & $262(72.0)$ & $<0.001$ \\
\hline MRA & $1534(64.8)$ & $1366(68.2)$ & $168(46.2)$ & $<0.001$ \\
\hline Loop diuretic & $2133(90.1)$ & $1836(91.6)$ & $297(81.6)$ & $<0.001$ \\
\hline Digoxin & $908(38.3)$ & $789(39.4)$ & $119(32.7)$ & 0.016 \\
\hline Ivabradine & 487 (20.6) & $471(23.5)$ & $16(4.4)$ & $<0.001$ \\
\hline CRT & $255(10.8)$ & $245(12.2)$ & $10(2.7)$ & $<0.001$ \\
\hline ICD & $356(15.0)$ & $334(16.7)$ & $22(6.0)$ & $<0.001$ \\
\hline
\end{tabular}

Table 1. Baseline demographic and clinical characteristics in patients with depressed and preserved ejection fraction (EF). Data are given as mean \pm standard deviation, median [interquartile range], or $n(\%) .{ }^{*} \mathrm{n}=2119$; ${ }^{\#} \mathrm{n}=2083 ;{ }^{\dagger} \mathrm{n}=1506 ;{ }^{5} \mathrm{n}=2361 ;{ }^{\ddagger} \mathrm{n}=2345 ;{ }^{9} \mathrm{n}=2350 ;{ }^{\ddagger} \mathrm{n}=1665$. ACEI, angiotensin-converting enzyme inhibitor; $\mathrm{ARB}$, angiotensin II receptor blocker; ARNI, angiotensin receptor-neprilysin inhibitor; BMI, body mass index; $\mathrm{CM}$, cardiomyopathy; COPD, chronic obstructive pulmonary disease; CRT, cardiac resynchronization therapy; HF, heart failure; ICD, implantable cardiac defibrillator; MAGGIC, Meta-analysis Global Group in Chronic Heart Failure; MRA, mineralocorticoid receptor antagonist; NT-proBNP, N-terminal pro-brain natriuretic peptide; NYHA, New York Heart Association. ${ }^{a}$ According to World Health Organization criteria $(<13 \mathrm{~g} / \mathrm{dL}$ in men and $<12 \mathrm{~g} / \mathrm{dL}$ in women). ${ }^{b}$ Estimated glomerular filtration rate (Chronic Kidney Disease-Epidemiology Collaboration equation) $<60 \mathrm{~mL} / \mathrm{min} / 1.73 \mathrm{~m}^{2}$. ${ }^{\mathrm{c}}$ Body mass index $\geq 30 \mathrm{~kg} / \mathrm{m}^{2}$. 


\begin{tabular}{|c|c|c|c|c|c|c|c|c|c|c|c|c|}
\hline & \multicolumn{6}{|c|}{ Depressed LVEF $(n=2004)$} & \multicolumn{6}{|c|}{ Preserved LVEF $(n=364)$} \\
\hline & $\begin{array}{l}\text { Quintile 1 } \\
(\mathrm{n}=423)\end{array}$ & $\begin{array}{l}\text { Quintile 2 } \\
(\mathrm{n}=412)\end{array}$ & $\begin{array}{l}\text { Quintile 3 } \\
(\mathrm{n}=417)\end{array}$ & $\begin{array}{l}\text { Quintile 4 } \\
(n=385)\end{array}$ & $\begin{array}{l}\text { Quintile 5 } \\
(\mathbf{n}=367)\end{array}$ & $P$ for trend & $\begin{array}{l}\text { Quintile 1 } \\
(\mathbf{n}=50)\end{array}$ & $\begin{array}{l}\text { Quintile 2 } \\
(\mathrm{n}=62)\end{array}$ & $\begin{array}{l}\text { Quintile 3 } \\
(\mathbf{n}=57)\end{array}$ & $\begin{array}{l}\text { Quintile } 4 \\
(n=89)\end{array}$ & $\begin{array}{l}\text { Quintile 5 } \\
(\mathrm{n}=106)\end{array}$ & $P$ for trend \\
\hline Age, years & $65.5 \pm 11.1$ & $66.9 \pm 12.6$ & $66.3 \pm 13.0$ & $66.3 \pm 12.7$ & $64.5 \pm 13.0$ & 0.25 & $70.9 \pm 9.9$ & $70.5 \pm 13.9$ & $68.9 \pm 16.1$ & $72.4 \pm 13.1$ & $64.9 \pm 16.0$ & 0.03 \\
\hline Male & $324(76.6)$ & $309(75.0)$ & $325(77.9)$ & $275(71.4)$ & $272(74.1)$ & 0.22 & $33(66.0)$ & $39(62.9)$ & $33(57.9)$ & $43(48.3)$ & $40(37.7)$ & $<0.001$ \\
\hline Caucasian & $421(99.5)$ & $405(98.3)$ & $398(95.4)$ & $369(95.8)$ & $348(94.8)$ & $<0.001$ & $50(100)$ & $62(100)$ & $56(98.2)$ & $87(97.8)$ & $102(96.2)$ & $<0.05$ \\
\hline Aetiology & & & & & & $<0.001$ & & & & & & $<0.001$ \\
\hline Ischaemic HD & $271(64.1)$ & $245(59.5)$ & $229(54.9)$ & $185(48.1)$ & $154(42.0)$ & & $11(22.0)$ & $12(19.4)$ & $7(12.3)$ & $13(14.6)$ & $14(13.2)$ & \\
\hline Dilated CM & $49(11.6)$ & $38(9.2)$ & $76(18.2)$ & $73(19.0)$ & $98(26.7)$ & & $1(2.0)$ & $0(0.0)$ & $2(3.5)$ & $4(4.5)$ & $4(3.8)$ & \\
\hline $\begin{array}{l}\text { Hypertensive } \\
\text { CM }\end{array}$ & $33(7.8)$ & $30(7.3)$ & $21(5.0)$ & $31(8.1)$ & $17(4.6)$ & & $15(30.0)$ & $17(27.4)$ & $17(29.8)$ & $24(27.0)$ & $14(13.2)$ & \\
\hline Alcohol CM & $28(6.6)$ & $22(5.3)$ & $24(5.8)$ & $15(3.9)$ & $22(6.0)$ & & $1(2.0)$ & $1(1.6)$ & $2(3.5)$ & $0(0.0)$ & $0(0.0)$ & \\
\hline $\begin{array}{l}\text { Drug-related } \\
\text { CM }\end{array}$ & $6(1.4)$ & $10(2.4)$ & $14(3.4)$ & $17(4.4)$ & $12(3.3)$ & & $1(2.0)$ & $1(1.6)$ & $1(1.8)$ & $2(2.2)$ & $1(0.9)$ & \\
\hline Valvular & $17(4.0)$ & $39(9.5)$ & $23(5.5)$ & $30(7.8)$ & $21(5.7)$ & & $16(32.0)$ & $23(37.1)$ & $14(24.6)$ & $20(22.5)$ & $12(11.3)$ & \\
\hline $\begin{array}{l}\text { Hypertrophic } \\
\text { CM }\end{array}$ & $0(0.0)$ & $0(0.0)$ & $1(0.2)$ & $1(0.3)$ & $4(1.1)$ & & $0(0.0)$ & $1(1.6)$ & $5(8.8)$ & $17(19.1)$ & $43(40.6)$ & \\
\hline Amyloidosis & $0(0.0)$ & $0(0.0)$ & $0(0.0)$ & $0(0.0)$ & $4(1.1)$ & & $0(0.0)$ & $0(0.0)$ & $4(7.0)$ & $3(3.4)$ & $2(1.9)$ & \\
\hline Other & $19(4.5)$ & $28(6.8)$ & $29(7.0)$ & $33(8.6)$ & $35(9.5)$ & & $5(10.0)$ & $7(11.3)$ & $5(8.8)$ & $6(6.7)$ & $16(15.1)$ & \\
\hline $\begin{array}{l}\text { HF duration, } \\
\text { months }\end{array}$ & 24 [2-54] & $4[1-36]$ & $6[2-42]$ & $6[2-28]$ & $3[1-19]$ & $<0.001$ & 14 [3-60] & 20 [5-48] & 14 [4-48] & $7[2-39]$ & $23[3-66]$ & 0.99 \\
\hline NYHA class & & & & & & $<0.001$ & & & & & & $<0.001$ \\
\hline I & $17(4.0)$ & $33(8.0)$ & $12(2.9)$ & $9(2.3)$ & $48(13.1)$ & & $1(2.0)$ & $3(4.8)$ & $5(8.8)$ & $8(9.0)$ & $30(28.3)$ & \\
\hline II & $216(51.1)$ & $254(61.7)$ & $327(78.4)$ & 287 (74.5) & $272(74.1)$ & & $19(38.0)$ & $34(54.8)$ & $32(56.1)$ & $44(49.4)$ & 49 (46.2) & \\
\hline III & $175(41.4)$ & $121(29.4)$ & 77 (18.5) & $87(22.6)$ & $47(12.8)$ & & $27(54.0)$ & $23(37.1)$ & $20(35.1)$ & $36(40.4)$ & $27(25.5)$ & \\
\hline IV & $15(3.5)$ & $4(1.0)$ & $1(0.2)$ & $2(0.5)$ & $0(0.0)$ & & $3(6.0)$ & $2(3.2)$ & $0(0.0)$ & $1(1.1)$ & $0(0.0)$ & \\
\hline LVEF, \% & $29.2 \pm 9.4$ & $29.3 \pm 8.5$ & $30.3 \pm 8.1$ & $31.7 \pm 8.4$ & $32.3 \pm 8.4$ & $<0.001$ & $61.6 \pm 8.6$ & $61.3 \pm 6.9$ & $60.0 \pm 7.8$ & $62.7 \pm 7.9$ & $62.7 \pm 7.8$ & 0.14 \\
\hline LVEDD, $\mathrm{mm}^{*}$ & $62.2 \pm 9.1$ & $61.9 \pm 8.3$ & $61.8 \pm 8.2$ & $61.1 \pm 7.8$ & $61.1 \pm 7.7$ & 0.01 & $50.5 \pm 7.3$ & $49.5 \pm 6.8$ & $49.4 \pm 8.1$ & $49.1 \pm 7.0$ & $47.2 \pm 5.8$ & 0.03 \\
\hline LVESD, $\mathrm{mm}^{*}$ & $49.0 \pm 10.5$ & $49.7 \pm 9.5$ & $50.0 \pm 9.4$ & $49.9 \pm 9.6$ & $49.4 \pm 9.1$ & 0.52 & $32.9 \pm 8.6$ & $33.8 \pm 6.8$ & $34.1 \pm 8.5$ & $32.1 \pm 7.6$ & $31.0 \pm 6.7$ & 0.20 \\
\hline $\begin{array}{l}\Delta \text { LVEF at } \\
1 \text { year }^{\dagger}\end{array}$ & $5.4 \pm 11.0$ & $7.8 \pm 11.7$ & $9.1 \pm 11.4$ & $10.5 \pm 12.2$ & $12.6 \pm 12.4$ & 0.001 & $-2.4 \pm 14.5$ & $0.6 \pm 9.6$ & $-0.6 \pm 7.7$ & $-0.2 \pm 8.1$ & $0.7 \pm 9.8$ & 0.21 \\
\hline Diabetes & $177(41.8)$ & $169(41.0)$ & $164(39.3)$ & $191(49.6)$ & $166(45.2)$ & 0.05 & $21(42.0)$ & $27(43.5)$ & $24(42.1)$ & $45(50.6)$ & $35(33.0)$ & 0.36 \\
\hline Hypertension & $234(55.3)$ & $243(59.0)$ & $264(63.3)$ & $272(70.6)$ & 245 (66.8) & $<0.001$ & $32(64.0)$ & 47 (75.8) & $43(75.4)$ & $66(74.2)$ & $59(55.7)$ & 0.09 \\
\hline COPD & $92(21.7)$ & $82(19.9)$ & $51(12.2)$ & $59(15.3)$ & $61(16.6)$ & 0.01 & $9(18.0)$ & $14(22.6)$ & $8(14.0)$ & $13(14.6)$ & $17(16.0)$ & 0.41 \\
\hline Anaemia $^{\S, a}$ & $179(42.3)$ & $198(48.3)$ & $181(43.4)$ & $201(52.6)$ & $138(37.7)$ & 0.63 & $29(58.0)$ & $34(54.8)$ & $27(47.4)$ & $55(62.5)$ & $30(28.3)$ & 0.001 \\
\hline $\begin{array}{l}\text { Renal } \\
\text { insufficiency } y^{\ddagger, b}\end{array}$ & $160(37.8)$ & $161(39.1)$ & $169(40.5)$ & $212(55.2)$ & $148(42.3)$ & 0.001 & $22(44.0)$ & $28(45.2)$ & $32(56.1)$ & $65(73.0)$ & $38(37.6)$ & 0.86 \\
\hline $\mathrm{AF} / \mathrm{FT}$ & $55(13.0)$ & $70(17.0)$ & $98(23.5)$ & $75(19.5)$ & $62(16.9)$ & 0.07 & $28(56.0)$ & $29(46.8)$ & $25(43.9)$ & $38(42.7)$ & $31(29.2)$ & 0.002 \\
\hline $\begin{array}{l}\text { Number of } \\
\text { comorbidities }\end{array}$ & $1.7 \pm 1.1$ & $1.8 \pm 1.1$ & $1.8 \pm 1.1$ & $2.1 \pm 1.2$ & $1.9 \pm 1.2$ & $<0.001$ & $2.2 \pm 1.1$ & $2.3 \pm 1.1$ & $2.3 \pm 1.2$ & $2.6 \pm 1.4$ & $1.7 \pm 1.4$ & 0.02 \\
\hline $\begin{array}{l}\text { 1-year death } \\
\text { risk (MAGGIC } \\
\text { score) }\end{array}$ & 13 [8-22] & 13 [9-23] & 13 [8-19] & 13 [9-21] & $11[8-18]$ & 0.001 & 12 [7-19] & 14 [8-23] & $12[7-21]$ & 19 [8-27] & $8[4-18]$ & 0.19 \\
\hline $\begin{array}{l}\text { 3-year death } \\
\text { risk (MAGGIC } \\
\text { score) }\end{array}$ & 32 [21-49] & 32 [23-49] & $32[21-43]$ & 32 [23-46] & 27 [19-40] & 0.001 & 29 [19-43] & 33 [21-49] & $29[18-46]$ & 43 [21-56] & 20 [11-40] & 0.10 \\
\hline BMI, $\mathrm{kg} / \mathrm{m}^{29}$ & 27 [24-31] & 27 [24-30] & 27 [24-29] & 27 [24-30] & 27 [24-30] & 0.58 & $28[24-33]$ & $28[25-33]$ & $27[24-31]$ & $27[24-31]$ & 28 [25-31] & 0.52 \\
\hline Obesity ${ }^{9, c}$ & $122(29.2)$ & $98(24.2)$ & $93(22.4))$ & $97(25.3)$ & $99(27.0)$ & 0.57 & $19(38.8)$ & $21(34.4)$ & $16(28.6)$ & $24(27.0)$ & $33(31.4)$ & 0.30 \\
\hline $\begin{array}{l}\text { NT-proBNP, } \\
\text { ng/L }\end{array}$ & - & $\begin{array}{l}1675 \\
{[713-3709]}\end{array}$ & $\begin{array}{l}1752 \\
{[688-4609]}\end{array}$ & \begin{tabular}{|l}
2085 \\
{$[941-4858]$}
\end{tabular} & $\begin{array}{l}1595 \\
{[720-3630]}\end{array}$ & 0.58 & - & $\begin{array}{l}785 \\
{[233-2010]}\end{array}$ & $\begin{array}{l}1329 \\
{[444-3047]}\end{array}$ & $\begin{array}{l}1800 \\
{[680-4180]}\end{array}$ & $\begin{array}{l}1007 \\
{[254-3709]}\end{array}$ & 0.21 \\
\hline \multicolumn{13}{|l|}{ Treatments } \\
\hline $\begin{array}{l}\text { ACEI/ARB/ } \\
\text { ARNI }\end{array}$ & $391(92.4)$ & $372(90.3)$ & $379(90.9)$ & $338(87.8)$ & 335 (91.3) & 0.24 & $38(76.0)$ & $48(77.4)$ & 37 (64.9) & $47(52.8)$ & $55(51.9)$ & $<0.001$ \\
\hline ARNI & $14(3.3)$ & $26(6.3)$ & $48(11.5)$ & $39(10.1)$ & $125(34.1)$ & $<0.001$ & $0(0.0)$ & $0(0.0)$ & $0(0.0)$ & $0(0.0)$ & $5(4.7)$ & 0.010 \\
\hline Beta-blocker & $351(83.0)$ & 377 (91.5) & 392 (94.0) & 368 (95.6) & 354 (96.5) & $<0.001$ & $28(56.0)$ & $43(69.4)$ & $46(80.7)$ & $67(75.3)$ & 78 (73.6) & $<0.05$ \\
\hline MRA & $247(58.4)$ & $244(59.2)$ & $267(64.0)$ & $295(76.6)$ & $313(85.3)$ & $<0.001$ & $29(58.0)$ & $27(43.5)$ & $29(50.9)$ & $44(49.4)$ & $39(36.8)$ & 0.04 \\
\hline Loop diuretic & 396 (93.6) & $379(92.0)$ & $372(89.2)$ & 354 (91.9) & 335 (91.3) & 0.26 & $46(92.0)$ & $59(95.2)$ & $52(91.2)$ & $77(86.5)$ & $63(59.4)$ & $<0.001$ \\
\hline Digoxin & $195(46.1)$ & $194(47.1)$ & $157(37.6)$ & $147(38.2)$ & $96(26.2)$ & $<0.001$ & $25(50.0)$ & $30(48.4)$ & $18(31.6)$ & $29(32.6)$ & $17(16.0)$ & $<0.001$ \\
\hline Ivabradine & $35(8.3)$ & $50(12.1)$ & $107(25.7)$ & $133(34.5)$ & $146(39.8)$ & $<0.001$ & $1(2.0)$ & $1(1.6)$ & $3(5.3)$ & $5(5.6)$ & $6(5.7)$ & 0.15 \\
\hline CRT & $35(8.3)$ & $39(9.5)$ & 61 (14.6) & $61(15.8)$ & $49(13.4)$ & 0.001 & $1(2.0)$ & $1(1.6)$ & $1(1.8)$ & $4(4.5)$ & $3(2.8)$ & 0.47 \\
\hline ICD & $59(13.9)$ & $61(14.8)$ & $81(19.4)$ & $71(18.4)$ & $62(16.9)$ & 0.09 & $1(2.0)$ & $0(0.0)$ & $3(5.3)$ & $10(11.2)$ & $8(7.5)$ & 0.02 \\
\hline Cardiac TR & $3(0.7)$ & $4(1.0)$ & $2(0.5)$ & $4(1.0)$ & 0 & 0.36 & 0 & 0 & $1(1.8)$ & 0 & 0 & - \\
\hline
\end{tabular}


Table 2. Baseline characteristics based on period of admission quintiles and left ventricular ejection fraction group. Data are given as mean \pm standard deviation, median [interquartile range], or $\mathrm{n}(\%){ }^{*} \mathrm{n}=2119$; ${ }^{\#} n=2083 ;{ }^{\dagger} n=1506 ;{ }^{\circledR} n=2361 ;{ }^{\ddagger} n=2345 ;{ }^{9} n=2350 ;{ }^{\sharp} n=1665$. ACEI, angiotensin-converting enzyme inhibitor; $\mathrm{AF} / \mathrm{FT}$, atrial fibrillation or flutter; ARB, angiotensin II receptor blocker; ARNI, angiotensin receptorneprilysin inhibitor; BMI, body mass index; CM, cardiomyopathy; COPD, chronic obstructive pulmonary disease; Cardiac TR, cardiac transplant; CRT, cardiac resynchronization therapy; HD, heart disease; HF, heart failure; ICD, implantable cardioverter-defibrillator; LVEF, left ventricular ejection fraction; MAGGIC, Meta-analysis Global Group in Chronic Heart Failure; MRA, mineralocorticoid receptor antagonist; NYHA, New York Heart Association; NT-proBNP, N-terminal pro-brain natriuretic peptide. a According to World Health Organization criteria $\left(<13 \mathrm{~g} / \mathrm{dL}\right.$ in men and $<12 \mathrm{~g} / \mathrm{dL}$ in women). ${ }^{\mathrm{b}}$ Estimated glomerular filtration rate (Chronic Kidney Disease-Epidemiology Collaboration equation) $<60 \mathrm{~mL} / \mathrm{min} / 1.73 \mathrm{~m}^{2}$. ${ }^{\mathrm{c}}$ Body mass index $\geq 30 \mathrm{~kg} / \mathrm{m}^{2}$.
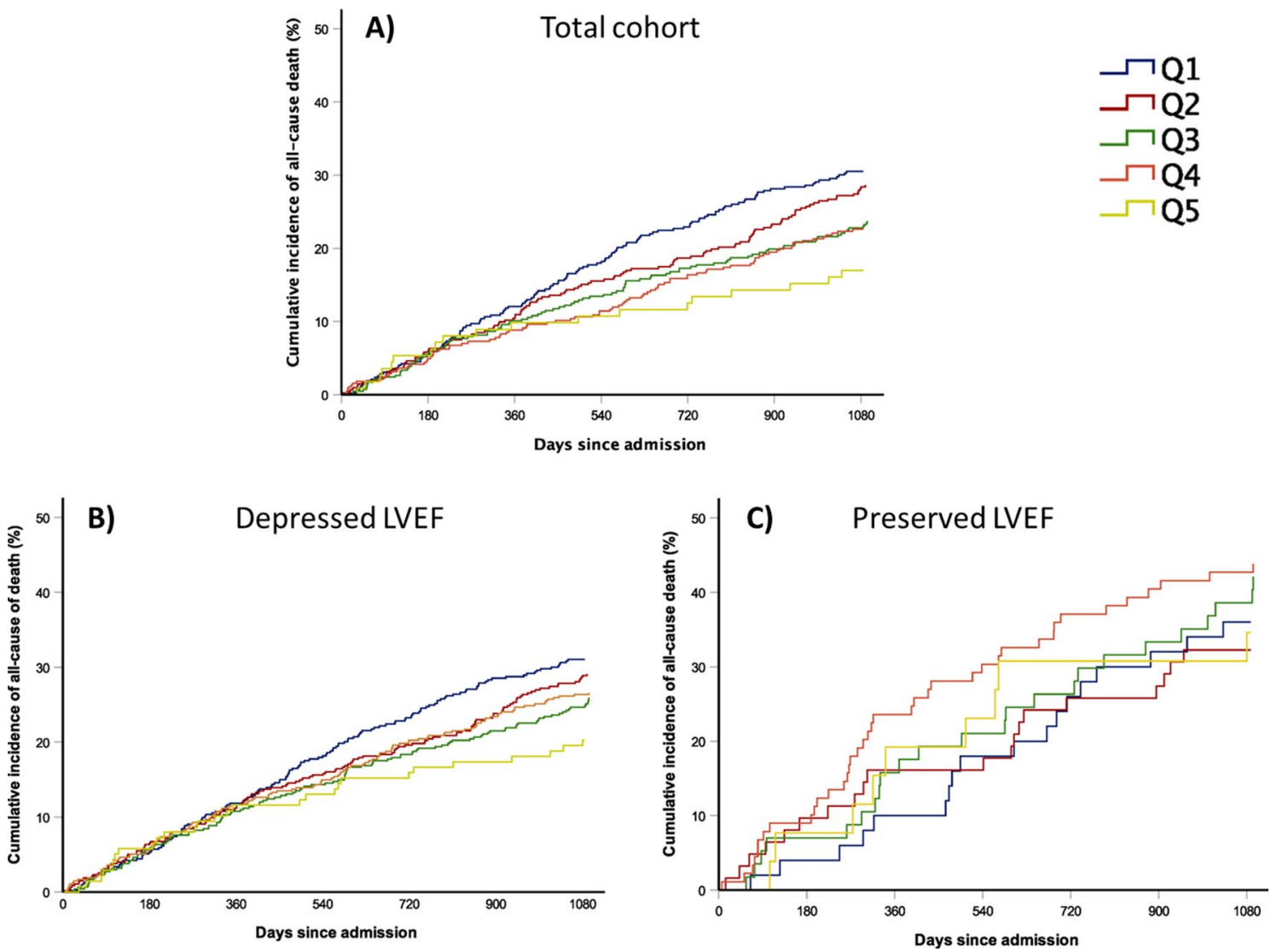

Figure 1. Cumulative incidence of death based on quintiles of period of admission; (A) Total cohort population; (B) Patients with depressed LVEF; (C) Patients with preserved LVEF. Q1, quintile 1, August 2001-August 2004; Q2, quintile 2, September 2004-November 2008; Q3, quintile 3, November 2008-June 2012; Q4, quintile 4, June 2012-October 2015; Q5, quintile 5, October 2015-September 2018.

Mortality trends according to period of admission quintiles. During the study period (median follow-up 4.14 years [IQR 1.9-7.7] for the total cohort; 2.9 [IQR 1.4-5.8] for HFpEF patients; and 4.4 [IQR 2.1-8.2] for patients with $\mathrm{LVEF}<50 \%), 1308$ patients $(55.2 \%)$ died, 708 (54.1\%) from cardiovascular causes (Supplementary Table S1 online). Supplementary Fig. 1 online shows Kaplan-Meier survival curves for the total cohort and the full follow-up at the HF Unit, according to the period of admission based on quintiles. Mortality was assessed in all patients at 1 year and in 2033 patients at 3 years. Figure 1 shows the cumulative incidence of death according to the period of admission for the total cohort and the two LVEF groups. In the overall HF population, no significant linear trend was observed with regards to all-cause $(\mathrm{p}=0.14)$ and cardiovascular death $(\mathrm{p}=0.10)$ at 1 year, but at 3 years, all-cause death rates fell progressively from $31.1 \%$ in Q1 to $20.3 \%$ in Q5 $(p=0.02)$ and cardiovascular mortality decreased significantly from $24.0 \%$ in Q1 to $13.0 \%$ in $\mathrm{Q} 5$ ( $\mathrm{p}=0.006$ ) (Fig. 2A,B). This linear trend remained significant for all-cause mortality after adjusting the multiple regression models for potential confounding factors (model $1, p=0.02$ ) and MAGGIC risk score (model 2, $\mathrm{p}<0.001$; Table 3). Similarly, this trend remained significant for cardiovascular mortality after adjusting for the same confounding factors (model 1, $\mathrm{p}=0.006$; Table 3) and MAGGIC risk score (model 2, $\mathrm{p}=0.04$; Table 3). As depicted in Fig. 2C,D, in patients 


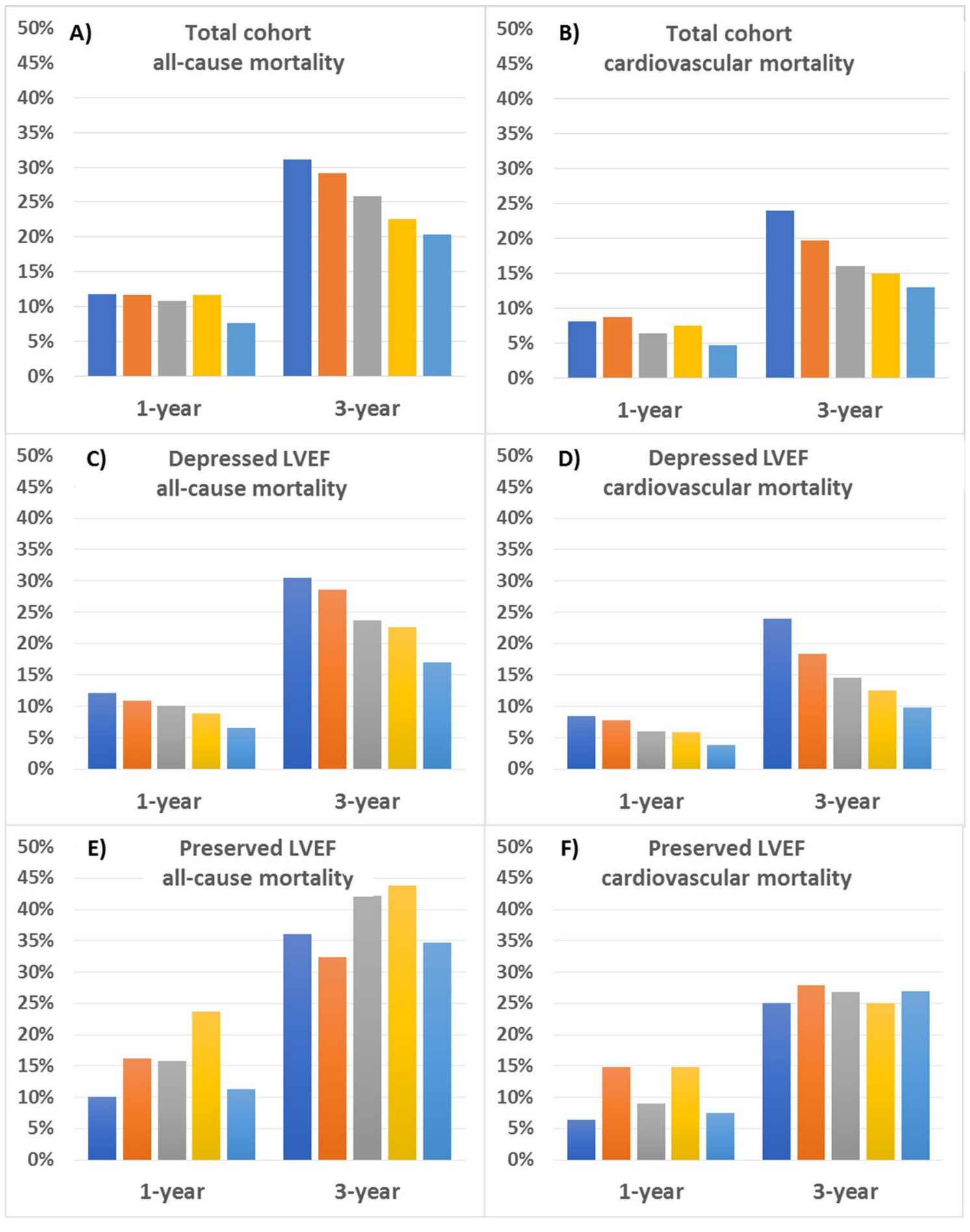

Q1 $\square$ Q2 $\square$ Q3 $\square$ Q4 $\square$ Q5

Figure 2. Trends in all-cause and cardiovascular mortality based on quintiles of period of admission. Upper panel, total cohort; middle panel, patients with depressed LVEF; bottom panel, patients with preserved LVEF. $(\mathbf{A}, \mathbf{C}, \mathbf{E})$ All-cause death; $(\mathbf{B}, \mathbf{D}, \mathbf{F})$ Cardiovascular death.

with depressed LVEF, we found a progressive decrease in both all-cause and cardiovascular death rates at 1 year (from 12.1\% in Q1 to 6.5\% in Q5, p=0.003; and from 8.4\% in Q1 to 3.8\% in Q5, p=0.007, respectively); and 3 years (from $30.5 \%$ in $\mathrm{Q} 1$ to $17.0 \%$ in $\mathrm{Q} 5, \mathrm{p}=0.003$; and from $23.9 \%$ in $\mathrm{Q} 1$ to $9.8 \%$ in $\mathrm{Q} 5, \mathrm{p}=0.003$, respectively). Almost all of these trends remained significant after adjusting for confounding factors and risk: all-cause mortality at 1 year (model $1, \mathrm{p}=0.003$; model 2, $\mathrm{p}=0.04$ ) and 3 years (model $1, \mathrm{p}=0.003$; model 2, $\mathrm{p}=0.03$ ), and cardiovascular mortality at 1 year (model $1, \mathrm{p}=0.007$; model $2, \mathrm{p}=0.05$ ) and 3 years (model $1, \mathrm{p}=0.003$; model $2, \mathrm{p}=0.02$; Table 3 ). Conversely, in the preserved LVEF subgroup, all-cause mortality rates at 1 and 3 years 


\begin{tabular}{|c|c|c|c|c|}
\hline & Beta-value & Standard error & $95 \% \mathrm{CI}$ & p-value \\
\hline \multicolumn{5}{|c|}{ Model 1} \\
\hline \multicolumn{5}{|c|}{ Total cohort $(n=2368)$} \\
\hline \multicolumn{5}{|c|}{ All-cause death } \\
\hline 3 years & -2.410 & 0.535 & $-4.114,-0.706$ & 0.02 \\
\hline \multicolumn{5}{|c|}{ Cardiovascular death } \\
\hline 3 years & -2.680 & 0.388 & $-3.916,-1.444$ & 0.006 \\
\hline \multicolumn{5}{|c|}{ LVEF < $50 \%(n=2004)$} \\
\hline \multicolumn{5}{|c|}{ All-cause death } \\
\hline 1 year & -1.330 & 0.155 & $-1.823,-0.837$ & 0.003 \\
\hline 3 years & -3.300 & 0.383 & $-4.520,-2.080$ & 0.003 \\
\hline \multicolumn{5}{|c|}{ Cardiovascular death } \\
\hline 1 year & -1.110 & 0.164 & $-1.632,-0.588$ & 0.007 \\
\hline 3 years & -3.410 & 0.394 & $-4.665,-2.155$ & 0.003 \\
\hline \multicolumn{5}{|c|}{ Model 2} \\
\hline \multicolumn{5}{|c|}{ Total cohort $(n=2368)$} \\
\hline \multicolumn{5}{|c|}{ All-cause death } \\
\hline 3 years & -1.906 & 0.026 & $-2.020,-1.792$ & $<0.001$ \\
\hline \multicolumn{5}{|c|}{ Cardiovascular death } \\
\hline 3 years & -2.719 & 0.538 & $-5.035,-0.402$ & 0.04 \\
\hline \multicolumn{5}{|c|}{ LVEF < 50\% $(n=2004)$} \\
\hline \multicolumn{5}{|c|}{ All-cause death } \\
\hline 1 year & -1.070 & 0.066 & $-1.352,-0.788$ & 0.004 \\
\hline 3 years & -2.860 & 0.497 & $-4.999,-0.721$ & 0.03 \\
\hline \multicolumn{5}{|c|}{ Cardiovascular death } \\
\hline 1 year & -0.930 & 0.220 & $-1.876,0.016$ & 0.05 \\
\hline 3 years & -3.810 & 0.554 & $-6.193,-1.427$ & 0.02 \\
\hline
\end{tabular}

Table 3. Data for periods of admission in multiple regression analyses. Model 1: Quintiles of admission period, age, sex, left ventricle ejection fraction, NYHA class III-IV, and number of comorbidities (diabetes, hypertension, anaemia, atrial fibrillation or flutter, chronic obstructive pulmonary disease). Multiple regression was stepwise analysis. Model 2: Quintiles of admission period and MAGGIC risk score at 1 and 3 years.

ranged from $10.0 \%$ to $11.3 \%$ and from $36.0 \%$ to $34.6 \%$, with no significant trend over time $(\mathrm{p}=0.63$ and $\mathrm{p}=0.65$, respectively; Fig. 2E-F). In the same subgroup of patients, we observed no significant trends in terms of cardiovascular mortality at $1(\mathrm{p}=0.88)$ or 3 years $(\mathrm{p}=0.86)$. In a sensitivity analysis, mortality was also explored using a preserved LVEF threshold of $\geq 40 \%$. Again, we did not find any significant trend in all-cause death (at 1 year, $\mathrm{p}=0.41$; at 3 years, $\mathrm{p}=0.98$ ) nor in cardiovascular mortality (at 1 year, $\mathrm{p}=0.45$; at 3 years, $\mathrm{p}=0.93$ ).

HF hospitalizations according to period of admission quintiles. No trend in the HF hospitalizations was found at 1 year $(\mathrm{p}=0.79)$ nor at 3 years $(\mathrm{p}=0.76)$ in the total cohort, although in those patients admitted in the last quintile period there were significantly less hospital admissions than in the other groups. The same figure was observed both for patients with depressed $(p=0.63$ and 0.53 , respectively) and for patients with preserved LVEF ( $\mathrm{p}=0.90$ and 0.90, respectively) (Supplementary Fig. 2).

\section{Discussion}

The main finding of our study is that mortality rates (both all-cause and cardiovascular) declined progressively at 3 years in a real-life ambulatory HF population over a 19-year period. In addition, patients with depressed LVEF had a significant reduction in all-cause and cardiovascular mortality at 1 and 3 years, but no significant trend in mortality was observed in patients with preserved LVEF.

Over the past 30 years, the survival of patients with HF has improved remarkably, in particular across trials including patients with HFrEF. Due to the introduction of drugs that inhibit the neurohormonal system and devices such as ICD and CRT, 1-year mortality rates in HFrEF patients have declined from approximately 15\% in the Studies of Left Ventricular Dysfunction (SOLVD) trial in 1991 to less than $10 \%$ in the recent Prospective Comparison of ARNI with ACEI to Determine Impact on Global Mortality and Morbidity in Heart Failure (PARADIGM-HF) and Dapagliflozin and Prevention of Adverse-outcomes in Heart Failure (DAPA-HF) trials ${ }^{1-10}$. However, patients included in randomized clinical trials (RCTs) usually differ from those that physicians manage every day in clinical practice; they are usually younger, more stable, have fewer comorbidities, higher adherence rates to therapy, and attend more follow-up visits than real-world patients ${ }^{17,18}$. In a recent systematic review of 118 trials published between 2001 and 2016 , Khan et al. found that $72 \%$ excluded patients with $\geq 1$ comorbid condition among dementia, anaemia, diabetes mellitus, severe or uncontrolled hypertension, chronic kidney 
disease (CKD), atrial fibrillation, chronic liver disease, stroke, cancer, and COPD ${ }^{19}$. CKD was the most common exclusion criterion ( $47 \%$ of trials). Regarding real-world data, in an Italian population-based database including 41,413 patients discharged from the hospital with a diagnosis of HF, the mean age was $78 \pm 11$ years and comorbidities, such as COPD, CKD, or cancer, accounted for up to $30 \%$ of cases $^{20}$. Furthermore, recent data from 207,984 patients in the Get-With-The Guidelines-Heart Failure-registry (GWTG-HF) indicate that the prevalence of $0,1,2$, and $\geq 3$ non-cardiovascular comorbidities is $18 \%, 30 \%, 27 \%$, and $25 \%$, respectively ${ }^{21}$. Another important issue that needs to be addressed is the significant gap in the use of guideline-directed medical therapy between RCTs and contemporary HF registries. As shown in the recent Change-the Management-ofPatients-with-Heart-Failure (CHAMP-HF) and the Chronisch-Hartfalen-ESC-richtlijn-Cardiologische-praktijk Kwaliteitsproject-HartFalen (CHECK-HF) registries, ACEI/ARB/ARNI, beta blockers and MRA were prescribed in up to $84 \%, 86 \%$ and $56 \%$ of eligible patients with HFrEF, respectively ${ }^{22,23}$.

These differences between RCTs and registries translate into an actual worse prognosis for real-world HF patients. A study from two U.S. community-based samples that included patients with HF from 1990 to 2009 reported that $67.4 \%$ of patients died at 5 years, and that mortality rates did not improve significantly across the decades considered ${ }^{24}$. Prognostic data from the GWTG-HF registry revealed that mortality in hospitalized HF patients can be as high as 75\% at the 5-year follow-up, regardless of the LVEF$^{12}$.

Despite being relatively young (66.4 \pm 12.9 years), patients admitted to our HF clinic had $1.9 \pm 1.2$ comorbidities (among diabetes, hypertension, anaemia, atrial fibrillation or flutter, COPD), and the overall prevalence of COPD, CKD, and anaemia was $17.1 \%, 44.1 \%$, and $45.4 \%$, respectively. With regards to prognosis, at the beginning of the study period (2001-2004), mortality rates were between those reported by RCTs and the rates from registries $(11.8 \%$ at 1 year and $31.1 \%$ at 3 years for all-cause death; $8.1 \%$ at 1 year and $24.0 \%$ at 3 years for cardiovascular death). Nevertheless, by 2015-2018, mortality rates were similar to those from contemporary RCTs, especially in the depressed LVEF subgroup (6.5\% at 1 year and $17 \%$ at 3 years for all-cause death; $3.8 \%$ at 1 year and $9.8 \%$ at 3 years for cardiovascular death). This could be related to the fact that our patients were progressively treated according to the trials demonstrating benefits of disease-modifying drugs and devices. In fact, in patients with $\mathrm{LVEF}<50 \%$, we found a significant increase in the prescription rates for neurohormonal antagonists, which reached $91.3 \%$ for ACEIs, ARBs, or ARNIs, 96.5\% for beta-blockers, and $85 \%$ for MRAs in the last quintile. Similarly, ICD and CRT rates increased significantly by $2015-2018$, to $16.9 \%$ and $13.4 \%$, respectively. On the other hand, patients with depressed LVEF admitted more recently reported less severe NYHA class symptoms, had higher LVEF, and lower MAGGIC risk score. Nevertheless, and very remarkable, the trend of lower mortality in these patients remained significant after adjusting for clinical confounders, such as age, sex, NYHA class, comorbidities, and LVEF, as well as the risk estimated by the MAGGIC score.

With respect to patients with preserved LVEF, survival did not improve over the last two decades in our cohort. Although a recent meta-analysis supports a possible beneficial effect of neurohormonal inhibitors in $\mathrm{HFpEF}^{25}$, no RCT has yet demonstrated a clear impact of any pharmacological treatment on survival in patients with $\mathrm{HFpEF}^{11}$. HFpEF is a very heterogeneous entity, and some authors have suggested phenomapping patients in order to classify them into different groups ${ }^{26}$. Personalized treatment based on this classification has been proposed $^{27}$, but to the best of our knowledge improved prognosis has not been demonstrated. In addition, patients with HFpEF admitted to our unit were older ( $69.1 \pm 14.5$ vs. $65.9 \pm 12.5$, p $<0.001)$ and had more comorbidities $(2.2 \pm 1.3$ vs. $1.8 \pm 1.2, \mathrm{p}<0.001)$ than the depressed LVEF subgroup, which indicates that the condition would be more difficult to influence with cardiac treatment. Nevertheless, patient age and comorbidities decreased significantly over time in our cohort, though no mortality reduction was observed. Finally, a recent review based on current evidence suggests that disease management programs for HF may improve survival and other outcomes in HFpEF patients, given that they are older and multi-morbid, and their management should not rely on a single-disease focus, but provide comprehensive care after geriatric assessment ${ }^{28}$. Our HF Unit is multidisciplinary and addresses the non-cardiac issues of HF patients, including comorbidities, geriatric evaluation, and rehabilitation. Despite this, no improvement in survival has been observed over the last two decades in patients with preserved LVEF. However, only one of the revised studies was focused on HFpEF, and it did not reduce all-cause or cardiovascular mortality ${ }^{29}$.

\section{Study limitations}

Our study has some limitations. First, the study cohort was a general HF population treated at a specific multidisciplinary HF clinic in a tertiary care hospital, with most patients referred from the Cardiology Department; thus, there was a predominance of relatively young men with HF of ischaemic aetiology and depressed LVEF, and the population was almost exclusively White. Therefore, we may not be able to fully extrapolate the results to other populations. Notably, a common treatment protocol was applied to all patients, limiting the possible bias introduced by different management strategies or treatment protocols. Second, the limited number of patients with preserved LVEF in our cohort makes the analysis of this subgroup less robust.

\section{Conclusion}

In a cohort of real-life ambulatory patients with HF of different aetiologies attended at a specialized HF clinic in a tertiary centre, mortality has progressively declined in patients with $\operatorname{LVEF}<50 \%$, but the same trend has not been observed in patients with preserved LVEF.

Received: 9 August 2020; Accepted: 7 December 2020

Published online: 12 January 2021 


\section{References}

1. SOLVD Investigators et al. Effect of enalapril on survival in patients with reduced left ventricular ejection fractions and congestive heart failure. N. Engl. J. Med. 325, 293-302 (1991).

2. Pitt, B. et al. The effect of spironolactone on morbidity and mortality in patients with severe heart failure Randomized Aldactone Evaluation Study Investigators. N. Engl. J. Med. 341, 709-717 (1999).

3. CIBIS-II Investigators and Committees. The Cardiac Insufficiency Bisoprolol Study II (CIBIS-II): a randomised trial. Lancet 353, 9-13 (1999).

4. Packer, M. et al. Effect of carvedilol on survival in severe chronic heart failure. N. Engl. J. Med. 344, 651-658 (2001).

5. Young, J. B. et al. Mortality and morbidity reduction with Candesartan in patients with chronic heart failure and left ventricular systolic dysfunction: results of the CHARM low-left ventricular ejection fraction trials. Circulation 110, 2618-2626 (2004).

6. Cleland, J. G. et al. The effect of cardiac resynchronization on morbidity and mortality in heart failure. N. Engl. J. Med. 352, 1539-1549 (2005).

7. Bardy, G. H. et al. Sudden Cardiac Death in Heart Failure Trial (SCD-HeFT) Investigators. Amiodarone or an implantable cardioverter-defibrillator for congestive heart failure. N. Engl. J. Med. 352, 225-237 (2005).

8. Zannad, F. et al. Eplerenone in patients with systolic heart failure and mild symptoms. N. Engl. J. Med. 364, 11-21 (2011)

9. McMurray, J. J. et al. Angiotensin-neprilysin inhibition versus enalapril in heart failure. N. Engl. J. Med. 371, 993-1004 (2014).

10. McMurray, J. J. V. et al. Dapagliflozin in patients with heart failure and reduced ejection fraction. N. Engl. J. Med. 381, 1995-2008 (2019).

11. Ponikowski, P. et al. 2016 ESC Guidelines for the diagnosis and treatment of acute and chronic heart failure: The Task Force for the diagnosis and treatment of acute and chronic heart failure of the European Society of Cardiology (ESC) Developed with the special contribution of the Heart Failure Association (HFA) of the ESC. Eur. Heart J. 37, 2129-2200 (2016).

12. Shah, K. S. et al. Heart failure with preserved, borderline, and reduced ejection fraction: 5-year outcomes. J. Am. Coll. Cardiol. 70, 2476-2486 (2017).

13. Parikh, K. S. et al. Heart failure with preserved ejection fraction expert panel report: current controversies and implications for clinical trials. JACC Heart Fail. 6, 619-632 (2018).

14. Solomon, S. D. et al. Angiotensin-neprilysin inhibition in heart failure with preserved ejection fraction. N. Engl. J. Med. 381, 1609-1620 (2019).

15. Zamora, E. et al. Estimated glomerular filtration rate and prognosis in heart failure: value of the Modification of Diet in Renal Disease Study-4, chronic kidney disease epidemiology collaboration, and cockroft-gault formulas. J. Am. Coll. Cardiol. 59, 1709-1715 (2012).

16. Lupón, J. et al. Dynamic trajectories of left ventricular ejection fraction in heart failure. J. Am. Coll. Cardiol. 72, 591-601 (2018).

17. Sharma, A. \& Ezekowitz, J. A. Similarities and differences in patient characteristics between heart failure registries versus clinical trials. Curr. Heart Fail. Rep. 10, 373-379 (2013).

18. Lund, L. H., Oldgren, J. \& James, S. Based pragmatic trials in heart failure: current experience and future directions. Curr. Heart Fail. Rep. 14, 59-70 (2017).

19. Khan, M. S. et al. Trends in prevalence of comorbidities in heart failure clinical. Eur. J. Heart Fail. 22, 1032-1042 (2020).

20. Maggioni, A. P. et al. The real-world evidence of heart failure: findings from 41413 patients of the ARNO database. Eur. J. Heart Fail. 18, 402-410 (2016).

21. Sharma, A. et al. Trends in noncardiovascular comorbidities among patients hospitalized for heart failure: insights from the get with the guidelines-heart failure registry. Circ. Heart Fail. 11, e004646 (2018).

22. Greene, S. J. et al. Medical therapy for heart failure with reduced ejection fraction: the CHAMP-HF Registry. J. Am. Coll. Cardiol. 72, 351-366 (2018).

23. Brunner-La Rocca, H. P. et al. Contemporary drug treatment of chronic heart failure with reduced ejection fraction: the CHECKHF registry. JACC Heart Fail. 7, 13-21 (2019).

24. Tsao, C. W. et al. Trends in the incidence of and mortality associated with heart failure with preserved and reduced ejection fraction. JACC Heart Fail. 6, 678-685 (2018).

25. Zheng, S. L. et al. Drug treatment effects on outcomes in heart failure with preserved ejection fraction: a systematic review and meta-analysis. Heart 104, 407-415 (2018).

26. Shah, S. J. et al. Phenomapping for novel classification of heart failure with preserved ejection fraction. Circulation 131, 269-279 (2015).

27. Shah, S. J. et al. Phenotype-specific treatment of heart failure with preserved ejection fraction: a multiorgan roadmap. Circulation 134, 73-90 (2016).

28. Kalogirou, F. et al. Heart failure disease management: a systematic review of effectiveness in heart failure with preserved ejection fraction. ESC Heart Fail. 7, 194-212 (2020).

29. Degryse, J. The impact of a nurse-led care programme on events and physical and psychosocial parameters in patients with heart failure with preserved ejection fraction: a randomized clinical trial in primary care in Russia. Eur. J. Gen. Pract. 16, 205-214 (2010).

\section{Aknowledgements}

We wish to thank the current nurses in the HF unit, Beatriz González, Carmen Rivas, Patricia Velayos, Ana Pulido, Eva Crespo and Violeta Díaz, and all the past nurses for data collection and their invaluable work in the unit. We also thank physicians Marta de Antonio and Pedro Moliner for their priceless work in the unit. We also wish to acknowledge Redes Temáticas de Investigación Cooperativa en Salud (RETICS) and Red Cardiovascular (RD12/0042/0047).

\section{Author contributions}

Conceptualization: J.L. and A.B.; Methodology: J.L., A.B., M.D., B.G.; Formal analysis: J.L., G.S, G.C.; Investigation: G.S., J.L, M.D., E.S., P.C, E.Z., G.C, J.S., C.D., M.I.T, M.B., S.A., N.A., J.N., A.B.; Writing—original draft preparation: G.S. and J.L.; Writing-review and editing: J.L., A.B.

Supervision: J.L. and A.B.; All authors have read and agreed to the published version of the manuscript.

\section{Competing interests}

The authors declare no competing interests.

\section{Additional information}

Supplementary Information is available for this paper at https://doi.org/10.1038/s41598-020-79926-3.

Correspondence and requests for materials should be addressed to A.B.-G. 
Reprints and permissions information is available at www.nature.com/reprints.

Publisher's note Springer Nature remains neutral with regard to jurisdictional claims in published maps and institutional affiliations.

(c) (i) Open Access This article is licensed under a Creative Commons Attribution 4.0 International License, which permits use, sharing, adaptation, distribution and reproduction in any medium or format, as long as you give appropriate credit to the original author(s) and the source, provide a link to the Creative Commons licence, and indicate if changes were made. The images or other third party material in this article are included in the article's Creative Commons licence, unless indicated otherwise in a credit line to the material. If material is not included in the article's Creative Commons licence and your intended use is not permitted by statutory regulation or exceeds the permitted use, you will need to obtain permission directly from the copyright holder. To view a copy of this licence, visit http://creativecommons.org/licenses/by/4.0/.

(C) The Author(s) 2021 